\title{
On the multiplicative structure of topological Hochschild homology
}

\author{
MORTEN BRUN \\ ZBIGNIEW FIEDOROWICZ \\ RAINER M VOGT
}

\begin{abstract}
We show that the topological Hochschild homology $\operatorname{THH}(R)$ of an $E_{n}$-ring spectrum $R$ is an $E_{n-1}$-ring spectrum. The proof is based on the fact that the tensor product of the operad $\mathcal{A}$ ss for monoid structures and the little $n$-cubes operad $\mathcal{C}_{n}$ is an $E_{n+1}$-operad, a result which is of independent interest.
\end{abstract}

55P43; $18 \mathrm{D} 50$

In 1993 Deligne asked whether the Hochschild cochain complex of an associative ring has a canonical action by the singular chains of the little 2-cubes operad. Affirmative answers for differential graded algebras in characteristic 0 have been found by Kontsevich and Soibelman [11], Tamarkin [15; 16] and Voronov [19]. A more general proof, which also applies to associative ring spectra is due to McClure and Smith [14]. In [10] Kontsevich extended Deligne's question: Does the Hochschild cochain complex of an $E_{n}$ differential graded algebra carry a canonical $E_{n+1}$-structure?

We consider the dual problem: Given a ring $R$ with additional structure, how much structure does the topological Hochschild homology $\mathrm{THH}(R)$ of $R$ inherit from $R$ ? The close connection of THH with algebraic $K$-theory and with structural questions in the category of spectra make multiplicative structures on THH desirable.

In his early work on topological Hochschild homology of functors with smash product, Bökstedt proved that THH of a commutative such functor is a commutative ring spectrum (unpublished). The discovery of associative, commutative and unital smash product functors of spectra simplified the definition of THH and the proof of the corresponding result for $E_{\infty}$-ring spectra considerably (see, for example, McClure, Schwänzl and Vogt [13]).

In this paper we morally prove the following:

Theorem A For $n \geq 2$, if $R$ is an $E_{n}$-ring spectrum then $\operatorname{THH}(R)$ is an $E_{n-1}$-ring spectrum. 
The same result has been obtained independently by Basterra and Mandell using different techniques [2].

Why "morally"? To define $\operatorname{THH}(R)$ we need $R$ to be a strictly associative spectrum. In general, $E_{n}$-structures do not have a strictly associative substructure. So we have to replace $R$ by an equivalent strictly associative ring spectrum $Y$, whose multiplication extends to an $E_{n}$-structure. Then the statement makes sense for $Y$. Here is a more precise reformulation of Theorem A.

Theorem B Let $R$ be an $E_{n}$-ring spectrum. Then there are $E_{n}$-ring spectra $X$ and $Y$ and maps of $E_{n}$-ring spectra

$$
Y \longleftarrow X \longrightarrow R
$$

which are homotopy equivalences of underlying spectra such that the $E_{n}$-structure on $Y$ extends a strictly associative ring structure and the topological Hochschild homology $\mathrm{THH}(Y)$ inherits an $E_{n-1}$-ring structure from $Y$.

Theorem $\mathrm{B}$ is an easy consequence of the universality of the $W$-construction of Boardman and Vogt $[5 ; 18]$ and an interchange result involving the operad structures of the operad $\mathcal{A} s s$ codifying monoid structures and the little $n$-cubes operad $\mathcal{C}_{n}$. The interchange is codified by the tensor product of operads (for terminology see 1.6). Our key result will be the following:

Theorem C $\mathcal{A} s s \otimes \mathcal{C}_{n}$ is an $E_{n+1}$-operad satisfying Condition 1.2 below.

The second author announced this theorem in [9] and sketched the main ideas for a proof. Here we will include a detailed proof by depicting the spaces $\left(\mathcal{A} s s \otimes \mathcal{C}_{n}\right)(k)$ as iterated colimits of diagrams of contractible spaces over posets. The diagrams of this iterated colimit combine to give a diagram over a Grothendieck construction, whose realization will turn out to be an $E_{n+1}$-operad.

Since this iterated colimit construction might be of use in other cases we give a formal definition in an appendix.

In this paper we will be working in the categories $\mathcal{T} o p, \mathcal{T} o p^{*}$ of (based) compactly generated spaces in the sense of Vogt [17] (often also called $k$-spaces), and $\mathcal{S} p$ of $S$-module spectra in the sense of Elmendorf, Kriz, Mandell and May [8]. Hence spectrum will always mean $S$-module spectrum.

Algebraic 83 Geometric Topology, Volume 7 (2007) 


\section{$1 \quad E_{n}-$ operads}

In this section we recall the basic definitions underlying the statement of Theorem $\mathrm{C}$.

Definition 1.1 An operad is a topologically enriched strict symmetric monoidal category $(\mathcal{B}, \oplus, 0)$ such that:

- $o b \mathcal{B}=\mathbb{N}$ and $m \oplus n=m+n$

- $\mathcal{B}(m, n)=\underset{r_{1}+\ldots+r_{n}=m}{\coprod_{1}} \mathcal{B}\left(r_{1}, 1\right) \times \ldots \times \mathcal{B}\left(r_{n}, 1\right) \times \Sigma_{r_{1}} \times \ldots \times \Sigma_{r_{n}} \Sigma_{m}$

Since the morphism spaces $\mathcal{B}(m, n)$ are determined by the spaces $\mathcal{B}(r, 1)$ we usually write $\mathcal{B}(r)$ for $\mathcal{B}(r, 1)$ and work with them. A map of operads $f: \mathcal{B} \rightarrow \mathcal{C}$ is a continuous strict symmetric monoidal functor such that $f(n)=n$ for all $n \in \mathbb{N}$. It is called a weak equivalence if $f: \mathcal{B}(n) \rightarrow \mathcal{C}(n)$ is a homotopy equivalence of spaces for all $n$. We call it a $\Sigma$-equivalence if these map are $\Sigma_{n}$-equivariant homotopy equivalences. An operad $\mathcal{B}$ is called $\Sigma$-free if $\mathcal{B}(r) \rightarrow \mathcal{B}(r) / \Sigma_{r}$ is a numerable principal $\Sigma_{r}$-bundle for all $r$.

For technical reasons we sometimes require the following condition:

Condition 1.2 $\{\mathrm{id}\} \subset \mathcal{B}(1)$ is a closed cofibration.

Definition 1.3 Let $\mathcal{C}_{n}$ denote the little $n$-cubes operad (see Boardman and Vogt [4, Chapter 2, Example 5]). An $E_{n}$-operad is an operad $\mathcal{B}$ for which there exists a sequence of $\Sigma$-equivalences of operads:

$$
\mathcal{B}=\mathcal{B}_{0} \stackrel{f_{0}}{\longrightarrow} \mathcal{B}_{1} \stackrel{f_{1}}{\longleftarrow} \cdots \longrightarrow \mathcal{B}_{r} \stackrel{f_{r}}{\longleftarrow} \mathcal{C}_{n}
$$

1.4 Let $\mathcal{O p r}$ denote the category of operads. In [5, Chapter III] Boardman and Vogt constructed a continuous functor

$$
W: \mathcal{O p r} \longrightarrow \mathcal{O} p r
$$

together with a natural transformation

$$
\varepsilon: W \longrightarrow \mathrm{Id}
$$

taking values in $\Sigma$-equivalences. It can be interpreted as a cofibrant replacement construction (see Vogt [18]). In particular, given a diagram of maps of operads

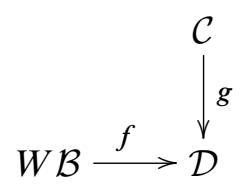

Algebraic $8 \mathcal{G}$ Geometric Topology, Volume 7 (2007) 
such that $\mathcal{B}$ satisfies Condition 1.2 and $g$ is a $\Sigma$-equivalence, then up to homotopy through operad maps there exists a lift $h: W \mathcal{B} \rightarrow \mathcal{C}$, and $h$ is unique up to homotopy through operad maps. If $\mathcal{B}$ is also $\Sigma$-free, the same holds if $g$ is only a weak equivalence [5, 3.17].

This implies the following proposition:

Proposition 1.5 Let $\mathcal{B}$ and $\mathcal{C}$ be $E_{n}$-operads such that $\mathcal{B}$ satisfies Condition 1.2. Let

$$
\mathcal{B}=\mathcal{B}_{0} \stackrel{f_{0}}{\longrightarrow} \mathcal{B}_{1} \stackrel{f_{1}}{\longleftarrow} \cdots \cdots \longrightarrow \mathcal{B}_{r} \stackrel{f_{r}}{\longleftarrow} \mathcal{C}
$$

be a sequence of weak equivalences connecting them. Then there is a diagram of weak equivalences

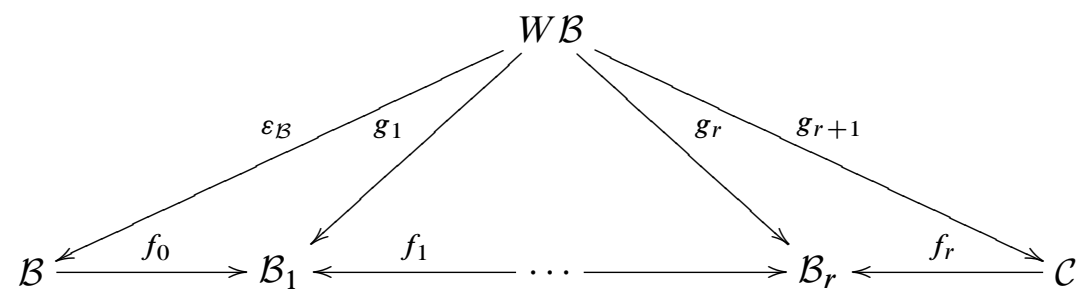

commuting up to homotopy through operad maps. The $g_{i}$ are unique up to homotopy through operad maps. In particular, taking $\mathcal{B}=\mathcal{C}_{n}$, there exists a weak equivalence $W \mathcal{C}_{n} \rightarrow \mathcal{C}$.

1.6 $E_{n}$-structures are closely related to $n$ interchanging $\left(E_{1}=A_{\infty}\right)$-structures. Let $\mathcal{C}$ and $\mathcal{D}$ be two operads and $X$ be an object having a $\mathcal{C}$ - and a $\mathcal{D}$-structure. These structures are said to interchange if for each $c \in \mathcal{C}(n)$ the operation

$$
c: X^{n} \longrightarrow X
$$

is a $\mathcal{D}$-homomorphism, or equivalently, for each $d \in \mathcal{D}(m)$ the operation

$$
d: X^{m} \longrightarrow X
$$

is a $\mathcal{C}$-homomorphism, ie the diagram

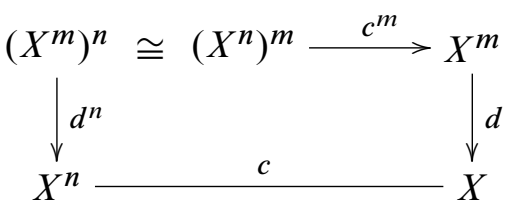

commutes for all $c \in \mathcal{C}(n)$ and all $d \in \mathcal{D}(m)$. 
The resulting structure on $X$ is codified by an operad $\mathcal{C} \otimes \mathcal{D}$ called the tensor product of $\mathcal{C}$ and $\mathcal{D}$. Formally, $\mathcal{C} \otimes \mathcal{D}$ is the quotient of the categorical sum $\mathcal{C} \oplus \mathcal{D}$ in $\mathcal{O p r}$ by factoring out relation (1-1). For more details see [5, page 40ff].

Theorem $C$ will be proved in Section 4. In Equation (4-4) we give an explicit chain of $\Sigma$-equivalences of operads connecting $\left(\mathcal{A} s s \otimes \mathcal{C}_{n}\right)$ with $\mathcal{C}_{n+1}$.

\section{Algebraic structures on spectra}

2.1 The category $\mathcal{S} p$ of spectra is enriched over $\mathcal{T} o p^{*}$ and complete and cocomplete in the enriched sense (for details see Elmendorf, Kriz, Mandell and May [8, Chapter VII]). If $K, L$ are based spaces and $M, N$ are spectra we have a natural isomorphism

$$
M \wedge(K \wedge L) \cong(M \wedge K) \wedge L
$$

and natural homeomorphisms

$$
\mathcal{S} p(M \wedge K, N) \cong \mathcal{T} \operatorname{op}^{*}(K, \mathcal{S} p(M, N)) \cong \mathcal{S} p(M, F(K, N))
$$

where $F(K, N)$ is the function spectrum. In particular,

$$
-\wedge K: \mathcal{S} p \longrightarrow \mathcal{S} p
$$

preserves colimits.

2.2 We can form the based topological endomorphism operad $\mathcal{E} n d_{M}$, given by

$$
\mathcal{E}_{n d}(n)=\mathcal{S} p\left(M^{\wedge n}, M\right)
$$

with the 0 -map as base point, where $M^{\wedge 0}=S$ is the sphere spectrum.

If $\mathcal{C}$ is any operad in $\mathcal{T}$ op , a $\mathcal{C}$-structure on $M$ is a based operad map

$$
\mathcal{C}_{+} \longrightarrow \mathcal{E} n d_{M}
$$

where $\mathcal{C}_{+}(n)=\mathcal{C}(n)_{+}=\mathcal{C}(n) \sqcup\{*\}$ with basepoint $*$. This transforms the topological operad $\mathcal{C}$ into a based topological operad $\mathcal{C}_{+}$; the monoidal structure in $\mathcal{T} o p^{*}$ is given by the smash product. Passing to adjoints a $\mathcal{C}$-structure on $M$ is given by a sequence of maps

$$
\mathcal{C}(n)_{+} \wedge \Sigma_{n} M^{\wedge n} \longrightarrow M, \quad n \in \mathbb{N}
$$

satisfying certain conditions due to the fact that $\mathcal{C}_{+} \rightarrow \mathcal{E} n d_{M}$ is a symmetric monoidal functor.

$M$ together with a given $\mathcal{C}$-structure is called a $\mathcal{C}$-algebra or $\mathcal{C}$-ring spectrum. 
To make sense of the interchange diagram (1-1) we have to give $M^{\wedge n}$ a $\mathcal{C}$-structure: If $M$ and $N$ are $\mathcal{C}$-algebras, then the canonical $\mathcal{C}$-algebra structure on $M \wedge N$ is given by the maps

$\mathcal{C}(n)_{+} \rightarrow(\mathcal{C}(n) \times \mathcal{C}(n))_{+}=\mathcal{C}(n)_{+} \wedge \mathcal{C}(n)_{+} \rightarrow \mathcal{E} n d_{M}(n) \wedge \mathcal{E} n d_{N}(n) \rightarrow \mathcal{E} n d_{M \wedge N}(n)$

where the first map is induced by the diagonal and the last by the smash product.

Finally we will need the proposition:

Proposition 2.3 If $M_{*}$ is a simplicial $\mathcal{C}$-algebra, then the realization $\left|M_{*}\right|$ inherits a $\mathcal{C}$-algebra structure.

This follows from the fact that $-\wedge \mathcal{C}(n)_{+}$preserves colimits. For details see $[8, \mathrm{X} .1 .3$, X. 1.4].

\section{THH of $E_{n}-$ ring spectra}

Definition 3.1 Let $\mathcal{B}$ and $\mathcal{C}$ be $E_{n}$-operads, let $R$ be a $\mathcal{B}$-algebra and $M$ be a $\mathcal{C}-$ algebra. An $E_{n}$-ring map from $R$ to $M$ is a pair $(\alpha, f)$ consisting of an operad map $\alpha: \mathcal{B} \rightarrow \mathcal{C}$ and a homomorphism $f: R \rightarrow M$ of $\mathcal{B}$-algebras, where the $\mathcal{B}$-structure on $M$ is the pulled back $\mathcal{C}$-structure.

3.2 Let $R \rightarrow M$ be an $E_{n}$-ring map between $E_{n}$-ring spectra. In view of Proposition 1.5 and Theorem $\mathrm{C}$ we may assume that it is a homomorphism of $W\left(\mathcal{A} s s \otimes \mathcal{C}_{n-1}\right)$ algebras.

For any operad $\mathcal{B}$ we have the free algebra functor $\mathbb{B}$ from spectra to $\mathcal{B}$-algebras defined by

$$
\mathbb{B}(X)=\bigvee_{n \geq 0} \mathcal{B}(n)_{+} \wedge_{\Sigma_{n}} X^{\wedge n}
$$

We now form the monadic bar constructions [8, Chapter XII] to obtain a diagram of $E_{n}$-ring spectra (here $\mathbb{W}(\mathcal{C})$ stands for the free algebra functor associated with the operad $W \mathcal{C}$ )

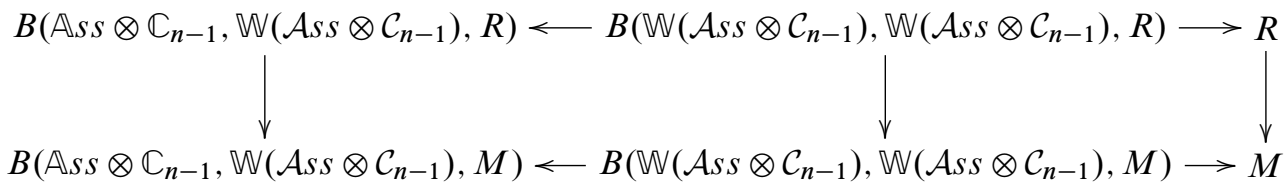

Algebraic ${ }^{3} \mathcal{G}$ Geometric Topology, Volume 7 (2007) 
and $E_{n}$-ring maps by Proposition 2.3. Since $\varepsilon: W\left(\mathcal{A} s s \otimes \mathcal{C}_{n-1}\right) \rightarrow \mathcal{A} s s \otimes \mathcal{C}_{n-1}$ is a weak $\Sigma$-equivalence, the horizontal maps are homotopy equivalences of spectra $[8, \mathrm{X}$. 2.4]. Let

$$
f: Y_{R} \longrightarrow Y_{M}
$$

denote the left vertical ( $\left.\mathcal{A} s s \otimes \mathcal{C}_{n-1}\right)$-algebra homomorphism. In particular, $f$ is a homomorphism of strictly associative, unital ring spectra, so that $Y_{M}$ is a $Y_{R}$-bimodule. We can form the topological Hochschild homology of $Y_{R}$ with coefficients in $Y_{M}$ :

3.3 Let $Q$ be a monoid in $\mathcal{S} p$ and $N$ a $Q$-bimodule. Then $\operatorname{THH}(Q ; N)$ is defined to be the realization of the simplicial spectrum

$$
[n] \longrightarrow \operatorname{THH}(Q ; N)_{n}=Q^{\wedge n} \wedge N
$$

with the well-known Hochschild boundary and degeneracy maps.

The inclusion of the 0 -skeleton defines a natural map

$$
\eta: N \longrightarrow \operatorname{THH}(Q ; N) \text {. }
$$

In our situation $\operatorname{THH}\left(Y_{R} ; Y_{M}\right)_{*}$ is a simplicial $\mathcal{C}_{n-1}$-algebra by the interchange relation (1-1). Hence $\operatorname{THH}\left(Y_{R} ; Y_{M}\right)$ is a $\mathcal{C}_{n-1}$-algebra and

$$
\eta: Y_{M} \longrightarrow \mathrm{THH}\left(Y_{R} ; Y_{M}\right)
$$

is a homomorphism of $\mathcal{C}_{n-1}$-algebras. We obtain the following generalization of Theorem B:

Theorem 3.4 Let $f: R \rightarrow M$ be an $E_{n}$-ring map between $E_{n}$-ring spectra. Then there is a commutative diagram of $E_{n}$-ring spectra and $E_{n}$-ring maps

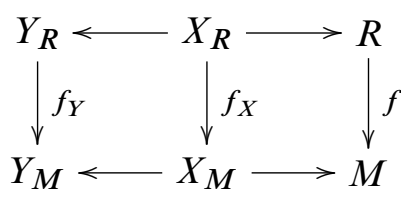

with the following properties:

(1) The horizontal maps are homotopy equivalences of spectra.

(2) $Y_{R}$ and $Y_{M}$ are $\left(\mathcal{A s s} \otimes \mathcal{C}_{n-1}\right)$-algebras and $f_{Y}$ is an $\left(\mathcal{A} s s \otimes \mathcal{C}_{n-1}\right)$-algebra homomorphism.

The second property implies that $\operatorname{THH}\left(Y_{R} ; Y_{M}\right)$ is a $\mathcal{C}_{n-1}$-ring spectrum, and the natural map $\eta: Y_{M} \rightarrow \operatorname{THH}\left(Y_{R} ; Y_{M}\right)$ is a $\mathcal{C}_{n-1}$-algebra homomorphism. 


\section{Proof of Theorem C}

In general analyzing the homotopy type of the tensor product of operads is an intractable problem. Our strategy is to use Berger's notion of a cellular decomposition of an operad over a poset operad [3]. Berger's cellular decomposition of $\mathcal{C}_{n}$ over the complete graphs operad $\mathcal{K}_{n}^{B}$ (definitions will follow) contains a minor flaw (see Remark 4.6) which can be corrected by modifying $\mathcal{K}_{n}^{B}$ slightly. We represent $\left(\mathcal{A s s} \otimes \mathcal{C}_{n}\right)(k)$ as the colimit of a diagram

$$
F_{k}: \mathcal{K}_{n+1}(k) \longrightarrow \mathcal{T} \text { op }
$$

of contractible spaces, indexed by the $k$-th space of the modification $\mathcal{K}_{n+1}$ of Berger's complete graphs operad $\mathcal{K}_{n+1}^{B}$, such that

- the diagrams are compatible with the operad structures of $\mathcal{K}_{n+1}$ and $\mathcal{A} s s \otimes \mathcal{C}_{n}$ in a sense we will make more precise below;

- the canonical map hocolim $F_{k} \rightarrow \operatorname{colim} F_{k}=\left(\mathcal{A s s} \otimes \mathcal{C}_{n}\right)(k)$ is a homotopy equivalence.

Then the collection of the hocolim $F_{k}$ forms an operad hocolim $F$ and we have a chain of weak equivalences of operads:

$$
\left|\mathcal{K}_{n+1}\right|=\operatorname{hocolim}_{\mathcal{K}_{n+1}} * \longleftarrow \operatorname{hocolim} F \longrightarrow \mathcal{A s s} \otimes \mathcal{C}_{n}
$$

Since the topological realization $\left|\mathcal{K}_{n+1}\right|$ of $\mathcal{K}_{n+1}$ is a $\Sigma$-free topological operad, so is hocolim $F$. We will show that $\left(\mathcal{A} s s \otimes \mathcal{C}_{n}\right)$ is $\Sigma$-free, hence both weak equivalences are $\Sigma$-equivalences. Moreover, Berger's argument in [3] applied to $\mathcal{K}_{n+1}$, as opposed to $\mathcal{K}_{n+1}^{B}$, proves that $\left|\mathcal{K}_{n+1}\right|$ is an $E_{n+1}$-operad, and we shall recapitulate the essential part of his argument in this section. Hence $\mathcal{A} s s \otimes \mathcal{C}_{n}$ is an $E_{n+1}$-operad, too.

4.1 The modified complete graphs operad $\mathcal{K}$ A coloring of the complete graph on the set of vertices $\{1,2,3, \ldots, k\}$ is an assignment of colors to each edge of the graph from the countable set of colors $\{1,2,3, \ldots\}$. A monochrome acyclic orientation of a colored complete graph on $k$ vertices is an assignment of direction to each edge of the graph such that no directed cycles of edges of the same color occur. The poset $\mathcal{K}(k)$ has as elements pairs $(\mu, \sigma)$, where $\mu$ is a coloring and $\sigma$ is a monochrome acyclic orientation of the complete graph on $k$ vertices. The order relation on $\mathcal{K}(k)$ is determined as follows: we say that $\left(\mu_{1}, \sigma_{1}\right) \leq\left(\mu_{2}, \sigma_{2}\right)$ if for any colored oriented edge $a \stackrel{i}{\longrightarrow} b$ in $\left(\mu_{1}, \sigma_{1}\right)$ the corresponding edge in $\left(\mu_{2}, \sigma_{2}\right)$ has orientation and coloring $a \stackrel{j}{\longrightarrow} b$ with $j \geq i$ or $b \stackrel{j}{\longrightarrow} a$ with $j>i$. The $n$-th filtration $\mathcal{K}_{n}(k)$ is the subposet of $\mathcal{K}(k)$ where the colorings are restricted to take values in the subset $\{1,2,3, \ldots, n\}$. 
The action of the symmetric group $\Sigma_{k}$ on $\mathcal{K}(k)$ is via permutation of the vertices. The composition

$$
\mathcal{K}(k) \times \mathcal{K}\left(m_{1}\right) \times \mathcal{K}\left(m_{2}\right) \times \cdots \times \mathcal{K}\left(m_{k}\right) \longrightarrow \mathcal{K}\left(m_{1}+m_{2}+\cdots+m_{k}\right)
$$

assigns to a tuple of orientations and colorings in $\mathcal{K}(k) \times \mathcal{K}\left(m_{1}\right) \times \mathcal{K}\left(m_{2}\right) \times \cdots \times \mathcal{K}\left(m_{k}\right)$ the orientation and coloring obtained by subdividing the set of $m_{1}+m_{2}+\cdots+m_{k}$ vertices into $k$ adjacent blocks containing $m_{1}, m_{2}, \ldots, m_{k}$ vertices respectively. The edges connecting vertices within the $i$-th block are oriented and colored according to the given element in $\mathcal{K}\left(m_{i}\right)$. The edges connecting vertices between blocks $i$ and $j$ are all oriented and colored according to the corresponding edge in the given element of $\mathcal{K}(k)$.

Berger's complete graphs operad $\mathcal{K}_{n}^{B}$ is the suboperad of $\mathcal{K}_{n}$ consisting of those oriented colored graphs which do not have any cycles, ie polychromatic cycles are also disallowed for elements in $\mathcal{K}_{n}^{B}(k)$.

4.2 Analysis of Ass $\otimes \mathcal{C}_{n} \quad$ By Boardman and Vogt [6, Theorem 5.5] the space $\left(\mathcal{A} s s \otimes \mathcal{C}_{n}\right)(k)$ is the quotient of $\Sigma_{k} \times \mathcal{C}_{n}(1)^{k}$ by the relation

$$
\left(\pi ; c_{1}, \ldots, c_{k}\right) \sim\left(\rho ; c_{1}, \ldots, c_{k}\right)
$$

if and only if $\pi^{-1}(i)<\pi^{-1}(j)$ and $\rho^{-1}(i)>\rho^{-1}(j)$ imply that $\left(c_{i}, c_{j}\right) \in \mathcal{C}_{n}(2) \subset$ $\mathcal{C}_{n}(1)^{2}$. (Recall that $\mathcal{C}_{n}(k)$ consists of the subspace of $k$-tuples of subcubes of the unit cube with disjoint interiors and thus can be naturally thought of as a subspace of $\mathcal{C}_{n}(1)^{k}$.) The element $\left(\sigma, c_{1}, \ldots, c_{k}\right) \in \Sigma_{k} \times \mathcal{C}_{n}(1)^{k}$ represents the operation $\left(x_{1}, \ldots, x_{k}\right) \mapsto$ $c_{\sigma 1}\left(x_{\sigma 1}\right) \cdot \ldots \cdot c_{\sigma k}\left(x_{\sigma k}\right)$, where $\cdot$ stands for the monoid multiplication. This also specifies the operad structure.

Observation 4.3 Since $\left(\mathcal{A s s} \otimes \mathcal{C}_{n}\right)(1)=\mathcal{C}_{n}(1)$ and $\mathcal{C}_{n}$ satisfies Condition 1.2, so does $\mathcal{A} s s \otimes \mathcal{C}_{n}$.

Since there is only one color for edges of elements in $\mathcal{K}_{1}(k)$, the operads $\mathcal{K}_{1}$ and $\mathcal{K}_{1}^{B}$ coincide. In particular, the elements in $\mathcal{K}_{1}(k)$ do not contain any cycles. An orientation with no cycles of the complete graph on the set of vertices $\{1,2, \ldots, k\}$ is a total ordering of $\{1,2, \ldots, k\}$, which in turn can be identified with a permutation of $\{1,2, \ldots, k\}$. Hence a representative of an element in $\left(\mathcal{A} s s \otimes \mathcal{C}_{n}\right)(k)$ can be identified with an oriented graph $\lambda \in \mathcal{K}_{1}(k)$ together with a labeling of the vertices by elements of $\mathcal{C}_{n}(1)$.

To take care of the relation (4-1) we enlarge the modified complete graphs operad: we allow complete graphs with partial monochrome acyclic orientations and partial 
colorings. Such graphs $\lambda^{\prime}$ are obtained from oriented colored graphs $\lambda \in \mathcal{K}(k)$ by choosing a subset $S$ of the set $E(k)$ of edges of $\lambda$ and forgetting the orientations and colors of all edges in $S$. Let $\widehat{\mathcal{K}}(k)$ denote the poset of all pairs $(\mu, \sigma)$, where $\mu$ is a partial coloring and $\sigma$ is a partial monochrome acyclic orientation of the complete graph on $k$ vertices obtained from some element in $\mathcal{K}(k)$. The order relation is defined as follows: $\left(\mu_{1}, \sigma_{1}\right) \leq\left(\mu_{2}, \sigma_{2}\right)$ if every uncolored unoriented edge in $\left(\mu_{1}, \sigma_{1}\right)$ is also uncolored unoriented in $\left(\mu_{2}, \sigma_{2}\right)$, and for any colored oriented edge $a \stackrel{i}{\longrightarrow} b$ in $\left(\mu_{1}, \sigma_{1}\right)$ the corresponding edge in $\left(\mu_{2}, \sigma_{2}\right)$ is either uncolored unoriented or has orientation and coloring $a \stackrel{j}{\longrightarrow} b$ with $j \geq i$ or $b \stackrel{j}{\longrightarrow} a$ with $j>i$.

The symmetric group actions and composition in $\widehat{\mathcal{K}}$, and the $n$-th filtration $\widehat{\mathcal{K}}_{n}$ are defined as in $\mathcal{K}$. We shall refer to $\widehat{\mathcal{K}}$ and its filtrations as the augmented complete graphs operad.

While the topological realization of $\mathcal{K}_{n}$ is an $E_{n}$-operad, this is not true for $\widehat{\mathcal{K}}_{n}$ : $\left|\widehat{\mathcal{K}}_{n}(k)\right|$ is equivariantly contractible to the $\Sigma_{k}$ fixed point specified by the complete graph on $\{1,2, \ldots, k\}$ with all its edges unoriented and uncolored, which is terminal in $\widehat{\mathcal{K}}_{n}(k)$.

If we now label the vertices of $\lambda \in \widehat{\mathcal{K}}_{1}(k)$ by elements in $\mathcal{C}_{n}(1)$ with the extra condition that the pair of labels $\left(c, c^{\prime}\right)$ of the end points of a nonoriented edge is an element of $\mathcal{C}_{n}(2)$, then $\lambda$ with its vertex labels $\left(c_{1}, \ldots, c_{k}\right)$ represents the equivalence class in $\left(\mathcal{A} s s \otimes \mathcal{C}_{n}\right)(k)$ of all $\left(\lambda^{\prime} ; c_{1}, \ldots, c_{k}\right)$, where $\lambda^{\prime} \in \mathcal{K}_{1}(k)$ is an element from which $\lambda$ can be obtained by forgetting orientations and colors. These labeled augmented complete graphs form an operad $\widehat{\mathcal{K}}_{1} \# \mathcal{C}_{n}$. Its composition is induced by the composition in $\widehat{\mathcal{K}}_{1}$ and the following labeling condition: if the $i$-th vertex of the element in $\widehat{\mathcal{K}}_{1}(k)$ has the label $a$ we compose the labels of the vertices of the elements in $\widehat{\mathcal{K}}_{1}\left(m_{i}\right)$ from the left with $a$. So $\left(\widehat{\mathcal{K}}_{1} \# \mathcal{C}_{n}\right)(k)$ is the disjoint union of all $A(\lambda), \lambda \in \widehat{\mathcal{K}}_{1}(k)$, where $A(\lambda) \subset \mathcal{C}_{n}(1)^{k}$ denotes the space of possible vertex labels of $\lambda$. We obtain that $\left(\mathcal{A} s s \otimes \mathcal{C}_{n}\right)(k)$ is a quotient of $\left(\widehat{\mathcal{K}}_{1} \# \mathcal{C}_{n}\right)(k)$. More precisely, the analysis of $\mathcal{A} s s \otimes \mathcal{C}_{n}$ of 4.2 can be restated as:

Lemma 4.4 $\left(\mathcal{A} s s \otimes \mathcal{C}_{n}\right)(k)$ is the colimit of the diagram

$$
A: \widehat{\mathcal{K}}_{1}(k)^{o p} \longrightarrow \mathcal{T} \text { op }, \quad \lambda \mapsto A(\lambda)
$$

where we consider each poset as a category with a morphism $\lambda_{1} \rightarrow \lambda_{2}$ whenever $\lambda_{1} \leq \lambda_{2}$.

Our next step is to depict $A(\lambda)$ as a colimit of contractible subspaces. Here $\mathcal{K}_{n+1}$ comes into the picture. Let $\lambda$ be a graph in $\widehat{\mathcal{K}}_{1}(k)$ and $S \subset E(k)$ the set of its colored 
edges. We define $T_{k}(\lambda)$ to be the subposet of $\widehat{\mathcal{K}}_{n}(k)$ of all $\lambda^{\prime}$ whose set of colored edges is precisely $E(k) \backslash S$.

We define $n$ strict order relations on $\mathcal{C}_{n}(1)$ as follows: Let $c_{1}, c_{2} \in \mathcal{C}_{n}(1)$ and let $\left(y_{1}, \ldots, y_{n}\right)$ be the highest corner of $c_{1}$ and $\left(x_{1}, \ldots, x_{n}\right)$ the lowest corner of $c_{2}$. For $1 \leq i \leq n$ we define

$$
c_{i}<_{i} c_{2} \quad \text { if and only if } y_{i} \leq x_{i} .
$$

For each $\mu \in \widehat{\mathcal{K}}_{n}(k)$ we define a closed subspace $H(\mu) \subset \mathcal{C}_{n}(1)^{k}$ by

$$
H(\mu)=\left\{\left(c_{1}, \ldots, c_{k}\right) \in \mathcal{C}_{n}(1)^{k} ; c_{p}<_{i} c_{q} \text { if } p \stackrel{i}{\rightarrow} q \text { in } \mu\right\},
$$

and we have a functor

$$
F_{k}(\lambda): T_{k}(\lambda) \longrightarrow \mathcal{T} \text { op }, \quad \lambda^{\prime} \mapsto \bigcup\left\{H(\mu) ; \mu \in T_{k}(\lambda) \mu \leq \lambda^{\prime}\right\}
$$

where the union is taken in $\mathcal{C}_{n}(1)^{k}$.

Lemma 4.5 $A(\lambda)=$ colim $F_{k}(\lambda)$. Moreover if $\mathcal{P}$ is an order ideal of $T_{k}(\lambda)$ (ie if $\alpha \in \mathcal{P}$, then each $\beta<\alpha$ is in $\mathcal{P}$ ), the colimit of the restriction to $\mathcal{P}$ is a subspace of $A(\lambda)$.

Proof Let $R(\lambda)=$ colim $F_{k}(\lambda)$. By construction, $A(\lambda)=\bigcup_{\lambda^{\prime} \in T(\lambda)} H\left(\lambda^{\prime}\right)$. Since the $H\left(\lambda^{\prime}\right)$ are closed subspaces of $A(\lambda)$, if suffices to show that the canonical map $p: R(\lambda) \rightarrow A(\lambda)$ is bijective. It is clearly surjective. So let $x \in H\left(\lambda_{1}\right) \cap H\left(\lambda_{2}\right) \subset A(\lambda)$. We need to show that $x \in H\left(\lambda_{1}\right)$ is related to $x \in H\left(\lambda_{2}\right)$ in the colimit $R(\lambda)$. Now $x=\left(c_{1}, \ldots, c_{k}\right) \in \mathcal{C}_{n}(1)^{k}$, and the little cubes $c_{1}, \ldots, c_{k}$ satisfy ordering conditions specified by $\lambda_{1}$ and $\lambda_{2}$. Define $\lambda_{3} \in \widehat{\mathcal{K}}_{n}(k)$ as follows: the edge between $p$ and $q$ obtains no color or orientation if the corresponding edges in $\lambda_{1}$ and $\lambda_{2}$ are not colored (note: by definition of $T_{k}(\lambda)$ an edge in $\lambda_{1}$ is not colored if and only if the corresponding edge in $\lambda_{2}$ is not colored). If both are colored, the corresponding edge in $\lambda_{3}$ obtains the color and orientation of the edge with the smaller color (if the colors agree, so do the orientations; this is forced by the ordering conditions for $c_{1}, \ldots, c_{k}$ ). The ordering conditions for $c_{1}, \ldots, c_{k}$ also imply that $\lambda_{3}$ does not have monochrome cycles. By construction, $\lambda_{3} \in T_{k}(\lambda)$ and $\lambda_{3} \leq \lambda_{1}$ and $\lambda_{3} \leq \lambda_{2}$, and $x \in H\left(\lambda_{3}\right)$. Hence $x \in H\left(\lambda_{1}\right)$ and $x \in H\left(\lambda_{2}\right)$ represent the same element in the colimit.

This argument also proves the second statement.

Remark 4.6 If $\lambda$ is the complete graph with no colors, then $T_{k}(\lambda)=\mathcal{K}_{n}(k)$, and Lemma 4.5 gives, in the terminology of Berger [3], a "cellular decomposition" of $\mathcal{C}_{n}(k)$ over $\mathcal{K}_{n}(k)$. In [3] Berger claimed that the same construction gives a cellular 
decomposition of $\mathcal{C}_{n}(k)$ over $\mathcal{K}_{n}^{B}(k)$ and used this to show that $\left|\mathcal{K}_{n}^{B}\right|$ is an $E_{n}$-operad. The following example illustrates that this construction does not give such a cellular decomposition over $\mathcal{K}_{n}^{B}$ :

Let $\left(c_{1}, c_{2}, c_{3}\right) \in \mathcal{C}_{3}(3)$ be the configuration with $c_{1}=\left[0, \frac{1}{2}\right] \times\left[\frac{2}{3}, 1\right] \times\left[0, \frac{1}{3}\right], c_{2}=$ $[0,1] \times\left[\frac{1}{3}, \frac{2}{3}\right] \times\left[\frac{1}{3}, \frac{2}{3}\right]$, and $c_{3}=\left[\frac{1}{2}, 1\right] \times\left[0, \frac{1}{3}\right] \times\left[\frac{2}{3}, 1\right]$. Over $\mathcal{K}_{3}^{B}$ this configuration lies in the interior of the cells $C_{\alpha}$ and $C_{\beta}$ where:
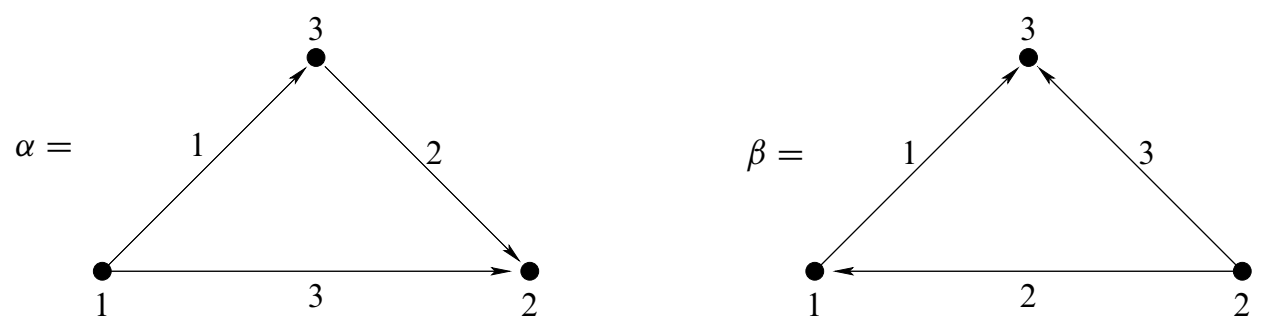

So the cells $C_{\alpha}$ and $C_{\beta}$ do not have disjoint interiors, which violates Berger's notion of a cellular decomposition. In contrast to $\mathcal{K}_{3}^{B}$, over $\mathcal{K}_{3}$, this configuration lies in $C_{\gamma}$ with

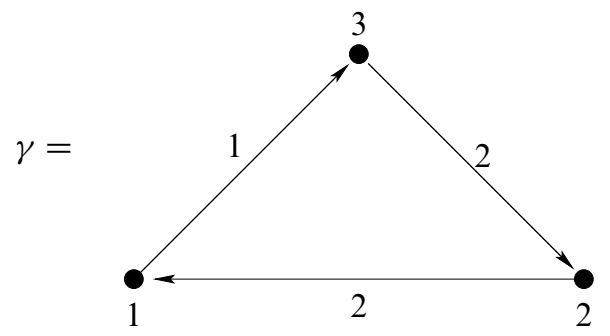

which is in the boundary of $C_{\alpha}$ and $C_{\beta}$. For definitions and terminology consult Berger [3].

We want to point out that results from [3] and [1] imply that $\left|\mathcal{K}_{n}^{B}\right|$ is an $E_{n}$-operad and that the inclusion $\left|\mathcal{K}_{n}^{B}\right| \subset\left|\mathcal{K}_{n}\right|$ is a $\Sigma$-equivalence.

The colimit decompositions of the $A(\lambda)$ are functorial with respect to the colimit decomposition of $\left(\mathcal{A s s} \otimes \mathcal{C}_{n}\right)(k)$ of Lemma 4.4 in the following sense: $T_{k}$ defines a functor:

$$
T_{k}: \widehat{\mathcal{K}}_{1}(k)^{\mathrm{op}} \longrightarrow \text { Posets, } \quad \lambda \mapsto T_{k}(\lambda)
$$

For a morphism $f: \lambda_{1} \rightarrow \lambda_{2}$ in $\widehat{\mathcal{K}}_{1}(k)^{\text {op }}$, ie $\lambda_{2} \leq \lambda_{1}$, we have a map of posets $T_{k}(f): T_{k}\left(\lambda_{1}\right) \longrightarrow T_{k}\left(\lambda_{2}\right)$ defined as follows: let $S_{i}$ be the set of uncolored unoriented edges of $\lambda_{i}$. Then $S_{2} \subset S_{1}$, and $E(k) \backslash S_{1}$ is the set of uncolored edges of any $\mu \in$ 
$T\left(\lambda_{1}\right)$. The map $T_{k}(f)$ sends $\mu$ to $\bar{\mu}$ obtained from $\mu$ by forgetting the orientations and colors of all edges in $E(k) \backslash S_{2}$. Moreover, $F_{k}\left(\lambda_{1}\right)(\mu) \subset F_{k}\left(\lambda_{2}\right)(\bar{\mu})$ because we have less order conditions on the cubes in $F_{k}\left(\lambda_{2}\right)(\bar{\mu})$. Hence the collection of functors $\left\{F_{k}(\lambda) ; \lambda \in \widehat{\mathcal{K}}_{1}(k)^{o p}\right\}$ is a $T_{k}$-indexed family of functors in the sense of Definition A.1 below and we can combine the diagrams to a diagram

$$
F_{k}: \widehat{\mathcal{K}}_{1}(k)^{\mathrm{op}} \int T_{k} \longrightarrow \mathcal{T} \text { op }, \quad(\lambda, \mu) \mapsto F_{k}(\lambda)(\mu)
$$

where $\widehat{\mathcal{K}}_{1}(k)^{\text {op }} \int T_{k}$ is the Grothendieck construction. Its objects are pairs $\left(\lambda, \lambda^{\prime}\right)$ with $\lambda \in \widehat{\mathcal{K}}_{1}(k)^{\text {op }}$ and $\lambda^{\prime} \in T_{k}(\lambda)$, and morphisms $(f, g):\left(\lambda_{1}, \lambda_{1}^{\prime}\right) \rightarrow\left(\lambda_{2}, \lambda_{2}^{\prime}\right)$ with $f: \lambda_{1} \rightarrow \lambda_{2}$ in $\widehat{\mathcal{K}}_{1}(k)^{\mathrm{op}}$, ie $\lambda_{2} \leq \lambda_{1}$ in $\widehat{\mathcal{K}}_{1}(k)$ and $g: T_{k}(f)\left(\lambda_{1}^{\prime}\right)=\bar{\lambda}_{1}^{\prime} \rightarrow \lambda_{2}^{\prime}$, ie $\overline{\lambda_{1}^{\prime}} \leq \lambda_{2}^{\prime}$, in $\widehat{\mathcal{K}}_{n}(k)$.

Lemma 4.7 $\left(\mathcal{A s s} \otimes \mathcal{C}_{n}\right)(k)=\operatorname{colim} F_{k}$.

Proof For $\lambda \in \widehat{\mathcal{K}}_{1}(k)^{\text {op }}$ let

$$
i_{\lambda}: T_{k}(\lambda) \longrightarrow \widehat{\mathcal{K}}_{1}(k)^{\mathrm{op}} \int T_{k}
$$

denote the inclusion. Then

$$
\operatorname{colim}_{\widehat{\mathcal{K}}_{1}(k)^{\mathrm{op}} \int T_{k}} F_{k}=\operatorname{colim}\left(\widehat{\mathcal{K}}_{1}(k)^{\mathrm{op}} \rightarrow \mathcal{T} \text { op }, \lambda \mapsto \operatorname{colim}_{T_{k}(\lambda)} F_{k} \circ i_{\lambda}\right) .
$$

Since $F_{k} \circ i_{\lambda}=F_{k}(\lambda)$, Lemma 4.4 and Lemma 4.5 imply the statement.

Lemma 4.8 There is an isomorphism of categories:

$$
\varphi: \mathcal{K}_{n+1}(k) \longrightarrow \widehat{\mathcal{K}}_{1}(k)^{\mathrm{op}} \int T_{k}
$$

Proof The isomorphism is defined by sending $\lambda \in \mathcal{K}_{n+1}(k)$ to $\left(\varphi_{1}(\lambda), \varphi_{2}(\lambda)\right)$ where $\varphi_{1}(\lambda)$ is obtained from $\lambda$ by replacing the color $n+1$ by the color 1 and by deleting colors and orientations of edges colored by any $i \leq n$, and $\varphi_{2}(\lambda)$ by forgetting colors and orientations of all edges colored by $n+1$. Observe that $\varphi_{2}(\lambda)$ is in $T_{k}\left(\varphi_{1}(\lambda)\right)$.

Lemma 4.9 For each $\lambda \in \mathcal{K}_{n+1}(k)$ the space $\left(F_{k} \circ \varphi\right)(\lambda)$ is contractible.

Proof $\left(F_{k} \circ \varphi\right)(\lambda)=\bigcup\left\{H(\mu) ; \mu \in T_{k}\left(\varphi_{1}(\lambda)\right), \mu \leq \varphi_{2}(\lambda)\right\}$. Berger has proved the contractability of $\bigcup\left\{H(\mu) ; \mu \in \mathcal{K}_{n}(k), \mu \leq \lambda\right\}$ with $\lambda \in \mathcal{K}_{n}^{B}(k)$ [3, Theorem 5.5]. The same argument applies to our situation. 
Lemma 4.10 The diagram $F_{k} \circ \varphi$ is Reedy-cofibrant, ie for all $\lambda \in \mathcal{K}_{n+1}(k)$

$$
\operatorname{colim}_{\mu<\lambda}\left(F_{k} \circ \varphi\right)(\mu) \longrightarrow\left(F_{k} \circ \varphi\right)(\lambda)
$$

is a closed cofibration.

Proof By Lemma 4.5, $\operatorname{colim}_{\mu<\lambda}\left(F_{k} \circ \varphi\right)(\mu)=\bigcup_{\mu<\lambda} H\left(\varphi_{2}(\mu)\right)$, so that we have to show that

$$
\bigcup_{\mu<\lambda} H\left(\varphi_{2}(\mu)\right) \subset \bigcup_{\mu \leq \lambda} H\left(\varphi_{2}(\mu)\right)
$$

is a closed cofibration. Let $\mu_{1}, \ldots, \mu_{s}$ be the predecessors of $\lambda$ and let $\lambda_{i}=\varphi_{2}\left(\mu_{i}\right)$, $\lambda^{\prime}=\varphi_{2}(\lambda)$. Since

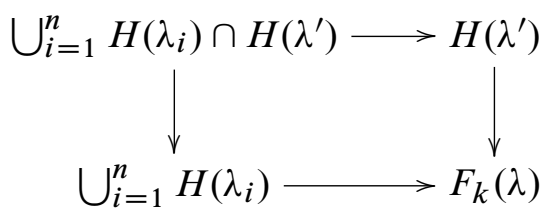

is a pushout, it suffices to show that

$$
\bigcup_{i=1}^{n} H\left(\lambda_{i}\right) \cap H\left(\lambda^{\prime}\right) \longrightarrow H\left(\lambda^{\prime}\right)
$$

is a closed cofibration. By Lillig's union theorem [12, Corollary 3] this holds if for any choice of objects $v_{1}, \ldots, v_{r} \in \widehat{\mathcal{K}}_{n}(k)$ the map

$$
H\left(v_{1}\right) \cap \ldots \cap H\left(v_{r}\right) \longrightarrow H\left(v_{r}\right)
$$

is a closed cofibration. A little cube $c \in \mathcal{C}_{n}(1)$ is determined by its lowest vertex $x=\left(x_{1}, \ldots, x_{n}\right)$ and its highest vertex $y=\left(y_{1}, \ldots, y_{n}\right)$. So $\mathcal{C}_{n}(1)^{k} \subset \mathbb{R}^{2 n k}$ is given by inequalities

$$
0 \leq x_{i j}<y_{i j} \leq 1 \quad i=1, \ldots, k, j=1, \ldots, n .
$$

The subspace $H(v) \subset \mathcal{C}_{n}(1)^{k}$ consists of elements satisfying additional nonstrict inequalities given by the ordering conditions.

Let $A \subset \mathbb{R}^{2 n k}$ be the subspace given by all inequalities determining $H\left(v_{1}\right) \cap \ldots \cap H\left(v_{r}\right)$ made nonstrict, and $X$ the corresponding space obtained from $H\left(v_{r}\right)$. Then $A \subset X$ clearly is a closed cofibration. Define $\tau: X \rightarrow[0,1]$ to be the product of all $\left(y_{i j}-x_{i j}\right)$ for which we have strict inequalities in $H\left(v_{1}\right) \cap \ldots \cap H\left(v_{r}\right)$ (they are the same as the ones in the list of $\left.H\left(v_{r}\right)\right)$, and let $\left.\left.V=\tau^{-1}(] 0,1\right]\right)$. Then by a result of Dold [7, Satz 1],

$$
V \cap A=H\left(v_{1}\right) \cap \ldots \cap H\left(v_{r}\right) \subset V=H\left(v_{r}\right)
$$

is a closed cofibration. 
Corollary 4.11 The canonical map hocolim $\left(F_{k} \circ \varphi\right) \rightarrow \operatorname{colim}\left(F_{k} \circ \varphi\right)=\left(\mathcal{A} s s \otimes \mathcal{C}_{n}\right)(k)$ is a homotopy equivalence.

For a proof see Balteanu, Fiedorowicz, Schwänzl and Vogt [1, Proposition 6.9], which shows that for a Reedy cofibrant diagram on a poset, the map from the homotopy colimit to the colimit is an equivalence.

Lemma 4.12 The operad $\mathcal{A} s s \otimes \mathcal{C}_{n}$ is $\Sigma$-free.

Proof By [6, Corollary 5.7] the $\Sigma_{k}$-action on $\left(\mathcal{A s s} \otimes \mathcal{C}_{n}\right)(k)$ is free. Since each space $\left(\mathcal{A} s s \otimes \mathcal{C}_{n}\right)(k)$ is Hausdorff and paracompact, the lemma follows.

The maps

$$
\left|\mathcal{K}_{n+1}(k)\right| \longleftarrow \operatorname{hocolim}\left(F_{k} \circ \varphi\right) \longrightarrow\left(\mathcal{A} s s \otimes \mathcal{C}_{n}\right)(k)
$$

assemble to $\Sigma$-equivalences of operads.

For each $k$ the maps are homotopy equivalences by Lemma 4.9 and Corollary 4.11. Both are equivariant homotopy equivalences since $\left|\mathcal{K}_{n+1}(k)\right|$ and $\left(\mathcal{A} s s \otimes \mathcal{C}_{n}\right)(k)$ are free $\Sigma_{k}$-spaces. It remains to prove that the collection of these maps form maps of operads. For this it suffices to show that

$$
H\left(\varphi_{2}(\lambda)\right) \circ\left(H\left(\varphi_{2}\left(\lambda_{1}\right)\right) \times \ldots \times H\left(\varphi_{2}\left(\lambda_{k}\right)\right)\right) \subset H\left(\varphi_{2}\left(\lambda \circ\left(\lambda_{1} \oplus \ldots \oplus \lambda_{k}\right)\right)\right)
$$

for $\lambda \in \mathcal{K}_{n+1}(k)$ and $\lambda_{i} \in \mathcal{K}_{n+1}\left(l_{i}\right), i=1, \ldots, k$. On the left side, composition is determined by the one in $\widehat{\mathcal{K}}_{1} \# \mathcal{C}_{n}$ ), on the right side we have composition in $\mathcal{K}_{n+1}$.

Condition (4-3) is a consequence of the following properties of our order relations on $\mathcal{C}_{n}(1)$ :

(i) $c_{1}<_{i} c_{2}$ if and only if $c_{3} \circ c_{1}<_{i} c_{3} \circ c_{2}$ for all $c_{3} \in \mathcal{C}_{n}(1)$

(ii) $c_{1}<_{i} c_{2} \Rightarrow c_{1} \circ c_{3}<_{i} c_{2} \circ c_{4}$ for all $c_{3}, c_{4} \in \mathcal{C}_{n}(1)$

Finally we show:

Lemma 4.13 $\left|\mathcal{K}_{n}\right|$ is an $E_{n}$-operad for each $n$.

Proof $\varphi\left(\mathcal{K}_{n}(k)\right) \subset \widehat{\mathcal{K}}_{1}(k)^{o p} \int T_{k}$ exactly consists of all those pairs $\left(\varphi_{1}(\lambda), \varphi_{2}(\lambda)\right)$ for which $\varphi_{1}(\lambda)$ does not have any colors. Hence $T_{k}\left(\varphi_{1}(\lambda)\right)=\mathcal{K}_{n}(k)$, and Diagram (4-2) restricts to a diagram of $\Sigma_{k}$-equivariant homotopy equivalences:

$$
\left|\mathcal{K}_{n}(k)\right| \longleftarrow \operatorname{hocolim}\left(F_{k} \circ\left(\varphi \mid \mathcal{K}_{n}(k)\right)\right) \longrightarrow \mathcal{C}_{n}(k)
$$


To distinguish between the $F_{k}$ for the various $n$ we denote $F_{k}$ above by $F_{k}^{(n)}$ and similarly for $\varphi$. Summarizing, there is an explicit chain of $\Sigma$-equivalences:

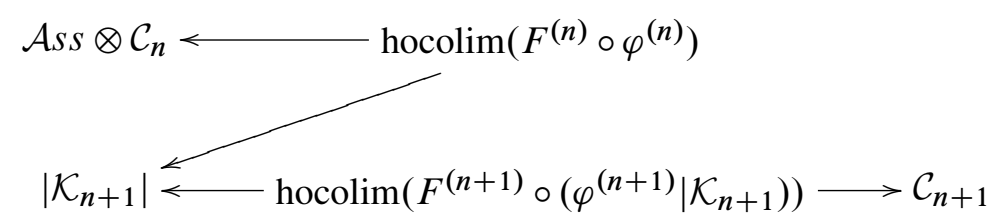

Together with Observation 4.3 this completes the proof of Theorem C.

\section{Appendix A Iterated colimits and Grothendieck's construc- tion}

Our description of $\left(\mathcal{A s s} \otimes \mathcal{C}_{n}\right)(k)$ as an iterated colimit is a special case of a more general situation, which may be of separate interest.

Let $F: \mathcal{A} \rightarrow \mathcal{C}$ at be any functor. Recall that the Grothendieck construction $\mathcal{A} \int F$ is the category whose objects are pairs $(A, B)$ with $A \in \operatorname{obj} \mathcal{A}$ and with $B \in \operatorname{obj} F(A)$. A morphism $\left(A_{1}, B_{1}\right) \longrightarrow\left(A_{2}, B_{2}\right)$ is a pair $(\alpha, \beta)$ where $\alpha: A_{1} \rightarrow A_{2}$ and $\beta: F(\alpha)\left(B_{1}\right) \rightarrow$ $B_{2}$.

Definition A.1 An $F$-indexed family of functors into a category $\mathcal{C}$ is a collection of functors

$$
\left\{G_{A}: F(A) \longrightarrow \mathcal{C}: A \in \operatorname{obj} \mathcal{A}\right\}
$$

and natural transformations

$$
\left\{\eta_{\alpha}: G_{A_{1}} \longrightarrow G_{A_{2}} F(\alpha): \alpha: A_{1} \rightarrow A_{2} \in \operatorname{mor} \mathcal{A}\right\}
$$

satisfying $\eta_{\mathrm{id}_{\mathrm{A}}}=\mathrm{id}_{\mathrm{G}_{\mathrm{A}}}$ for all $A \in \operatorname{obj} \mathcal{A}$ and satisfying the following associativity conditions

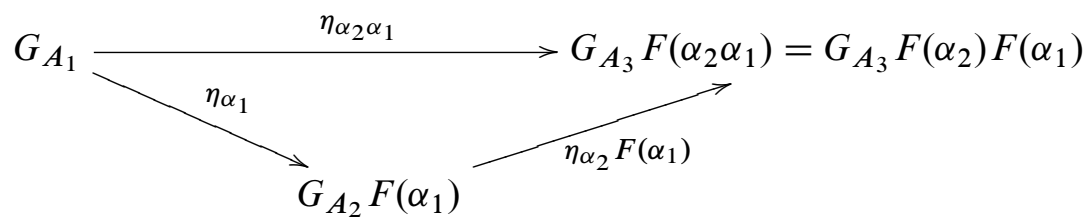

for any composable pair of morphisms in $\mathcal{A}$ :

$$
A_{1} \stackrel{\alpha_{1}}{\longrightarrow} A_{2} \stackrel{\alpha_{2}}{\longrightarrow} A_{3}
$$


An $F$-indexed family of functors determines a functor $G \int F: \mathcal{A} \int F \longrightarrow \mathcal{C}$ given on objects by $G \int F(A, B)=G_{A}(B)$ and on morphisms $(\alpha, \beta):\left(A_{1}, B_{1}\right) \longrightarrow\left(A_{2}, B_{2}\right)$ by

$$
G_{A_{1}}\left(B_{1}\right) \stackrel{\eta_{\alpha}}{\longrightarrow} G_{A_{2}} F(\alpha)\left(B_{1}\right) \stackrel{G_{A_{2}}(\beta)}{\longrightarrow} G_{A_{2}}\left(B_{2}\right) .
$$

Now suppose $\mathcal{C}$ is a category with small colimits. Then the natural transformations $\eta_{\alpha}$ induce a functor $\mathcal{A} \rightarrow \mathcal{C}$, which takes an object $A$ to $\operatorname{colim}_{F(A)} G_{A}$. We then have:

Proposition A.2 $\operatorname{colim}_{A \in \mathrm{obj} \mathcal{A}}\left(\operatorname{colim}_{F(A)} G_{A}\right) \cong \operatorname{colim}_{\mathcal{A} \int F} G \int F$

The proof is straight forward.

\section{References}

[1] C Balteanu, Z Fiedorowicz, R Schwänzl, R Vogt, Iterated monoidal categories, Adv. Math. 176 (2003) 277-349 MR1982884

[2] M Basterra, M Mandell, The multiplication on $M U$ and $B P$, to appear

[3] C Berger, Combinatorial models for real configuration spaces and $E_{n}$-operads, from: "Operads: Proceedings of Renaissance Conferences (Hartford, CT/Luminy, 1995)", (J-L Loday, J D Stasheff, A A Voronov, editors), Contemp. Math. 202, Amer. Math. Soc., Providence, RI (1997) 37-52 MR1436916

[4] J M Boardman, R M Vogt, Homotopy-everything H-spaces, Bull. Amer. Math. Soc. 74 (1968) 1117-1122 MR0236922

[5] J M Boardman, R M Vogt, Homotopy invariant algebraic structures on topological spaces, Lecture Notes in Mathematics 347, Springer, Berlin (1973) MR0420609

[6] J M Boardman, R M Vogt, Tensor products of theories, application to infinite loop spaces, J. Pure Appl. Algebra 14 (1979) 117-129 MR524181

[7] A Dold, Die Homotopieerweiterungseigenschaft (= HEP) ist eine lokale Eigenschaft, Invent. Math. 6 (1968) 185-189 MR0246292

[8] A D Elmendorf, I Kriz, M A Mandell, J P May, Rings, modules, and algebras in stable homotopy theory, Mathematical Surveys and Monographs 47, American Mathematical Society, Providence, RI (1997) MR1417719 With an appendix by M. Cole

[9] Z Fiedorowicz, Constructions of $E_{n}$ Operads, from: "Proceedings of the Workshop on Operads", University of Bielefeld (1999) 34-55

[10] M Kontsevich, Operads and motives in deformation quantization, Lett. Math. Phys. 48 (1999) 35-72 MR1718044Moshé Flato (1937-1998)

[11] M Kontsevich, Y Soibelman, Deformations of algebras over operads and the Deligne conjecture, from: "Conférence Moshé Flato 1999, Vol. I (Dijon)", Math. Phys. Stud. 21, Kluwer Acad. Publ., Dordrecht (2000) 255-307 MR1805894 
[12] J Lillig, A union theorem for cofibrations, Arch. Math. (Basel) 24 (1973) 410-415 MR0334193

[13] J McClure, R Schwänzl, R Vogt, $\mathrm{THH}(R) \cong R \otimes S^{1}$ for $E_{\infty}$ ring spectra, J. Pure Appl. Algebra 121 (1997) 137-159 MR1473888

[14] J E McClure, J H Smith, A solution of Deligne's Hochschild cohomology conjecture, from: "Recent progress in homotopy theory (Baltimore, MD, 2000)", Contemp. Math. 293, Amer. Math. Soc., Providence, RI (2002) 153-193 MR1890736

[15] DE Tamarkin, Another proof of $M$. Kontsevich formality theorem arXiv: math.QA/9803025

[16] D E Tamarkin, Formality of chain operad of little discs, Lett. Math. Phys. 66 (2003) 65-72 MR2064592

[17] R M Vogt, Convenient categories of topological spaces for homotopy theory, Arch. Math. (Basel) 22 (1971) 545-555 MR0300277

[18] R M Vogt, Cofibrant operads and universal $E_{\infty}$ operads, Topology Appl. 133 (2003) 69-87 MR1996461

[19] A A Voronov, Homotopy Gerstenhaber algebras, from: "Conférence Moshé Flato 1999, Vol. II (Dijon)", Math. Phys. Stud. 22, Kluwer Acad. Publ., Dordrecht (2000) 307-331 MR1805923

Department of Mathematics, University of Bergen

Johs. Brunsgt. 12, N-5008 Bergen, Norway

Department of Mathematics, The Ohio State University

Columbus OH 43210-1174, USA

Universität Osnabrück

Fachbereich Mathematik/Informatik, Albrechtstr. 28a, 49069 Osnabrück, Germany

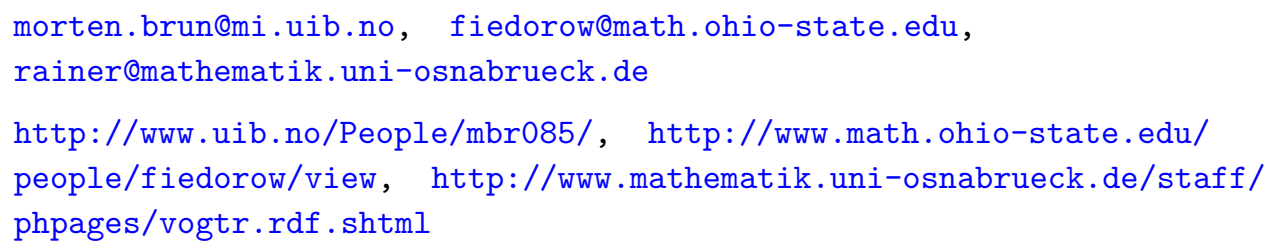

Received: 25 June 2007 Revised: 10 September 2007 